Ozga Alicja, Obuchowska Aleksandra, Standyło Arkadiusz, Wójcik Justyna, Skorupski Pawel. Laparoscopic myomectomy in the treatment of large myomas - unnecessary risk or effective method? Journal of Education, Health and Sport. 2020;10(9):860-867. eISSN 2391-8306. DOI http://dx.doi.org/10.12775/JEHS.2020.10.09.104

https://apcz.umk.pl/czasopisma/index.php/JEHS/article/view/JEHS.2020.10.09.104

https://zenodo.org/record/4054998

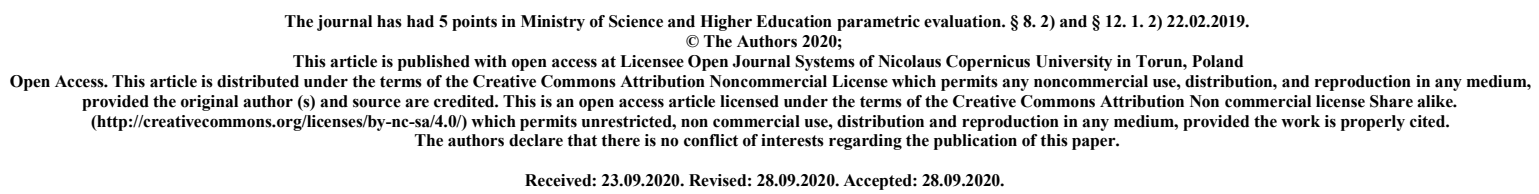

\title{
Laparoscopic myomectomy in the treatment of large myomas - unnecessary risk or effective method?
}

\author{
Alicja Ozga ${ }^{1 \mathrm{a}}$, Aleksandra Obuchowska ${ }^{1 \mathrm{~b}}$, Arkadiusz Standyło ${ }^{1 \mathrm{c}}$, Justyna Wójcik ${ }^{1 \mathrm{~d}}$, \\ Pawel Skorupski ${ }^{2 e}$
}

${ }^{1}$ Student Scientific Group at the $2^{\text {nd }}$ Chair and Department of Gynecology, Medical University of Lublin, Lublin, Poland

${ }^{2} 2^{\text {nd }}$ Chair and Department of Gynecology, Medical University of Lublin, Lublin, Poland

aaozga1@gmail.com; https://orcid.org/0000-0003-1291-905X

baobuchowska12@gmail.com; https://orcid.org/0000-0003-0464-2695

ca.standylo@gmail.com; https://orcid.org/0000-0002-5154-4759

d justynawojcik455@gmail.com; https://orcid.org/0000-0001-7163-6784

epawskor@tlen.pl;

\section{Corresponding author:}

Alicja Ozga

Jaczewskiego 8 Street

20-954 Lublin, Poland

e-mail: aozga1@gmail.com 


\begin{abstract}
Introduction:

Uterine myomas are the most common benign tumors of the uterus. Most of the myomas are asymptomatic and do not require medical intervention, however if they do, a surgical approach is still the mainstay of the therapy. Myomectomy is one of the surgical methods of removing myomas for those who want to preserve fertility. Nowadays, surgical techniques are improved and less invasive methods like laparoscopic myomectomy (LM) were developed. However, the safety and the efficacy of LM is well documented in the case of average size fibroids. Laparoscopic approach in larger uterine tumors (diameter $>10 \mathrm{~cm}$ ) can be controversial and can create a challenge due to possible difficulties.
\end{abstract}

\title{
Purpose:
}

Evaluation of effectiveness, technical challenges and complications of LM in treatment of large myomas.

\section{Materials and methods:}

We performed search of Pubmed, Springer Link and ResearchGate databases.

\section{Results:}

Systematic analyses qualified for this review suggested that despite many possible complications and difficulties, a method can be applied successfully by an experienced specialist. However, LM in large myomas treatment is still rare and data on this subject is still limited.

\section{Conclusions:}

Laparoscopic myomectomy can be an alternative method in the treatment of large fibroids. Laparoscopic surgery of large myomas LM can be challenging and the effectiveness of a such treatment depends on the surgeon's skills. The method is also useful in women who consider pregnancy.

Key words: laparoscopic; myomectomy; myoma; tumor 


\section{Introduction:}

Uterine myomas (fibromas, leiomyomas) are benign tumors that arise from the myometrium. They are composed of smooth muscle tissue and affect about 25 to $30 \%$ women in the reproductive age ${ }^{1}$. The precise cause of uterine fibroids is unknown, but some risk factors were identifies and include genetic predispositions, obesity, environmental factors, diet and race.

A crucial process in the diagnostics for uterine myomas is bimanual pelvic examination, ultrasonography and MRI (magnetic resonance imaging). ${ }^{2}$ MRI when available is also preferred for planning a surgical approach. Most cases of are asymptomatic, however symptoms depend on their size, degenerative changes and location ${ }^{3}$. Tumors are commonly multiple, though they can grow anywhere the most frequent location is myometrium. They can reach is $>10 \mathrm{~cm}$, however most of the myomas are smaller and do not require intervention. A myoma is considered as large when its diameter exceeds $10 \mathrm{~cm}$.

It is believed that treatment is indicated only when fibroids cause symptoms for example painful menstruation, menometrorrhagia, serious hemorrhage, infertility or repeated miscarriages ${ }^{4}$. Management of fibromas is based on pharmacotherapy, surgery or a combination of these approaches. Patients with anemia or large and multiple fibroids can be treated prior to the surgery with gonadotropin-releasing hormone analogs or ulipristal acetate. Such regimen effectively reduces tumor size and facilitates surgical procedure ${ }^{5}$. One has to remember that current treatment rely mostly on surgical methods, however a therapy must be individualized. An approach depends on the myomas' location, number and size and willingness of the patient to retain fertility.

It is said that hysterectomy is the most effective method of symptomatic fibroids management but clearly it is not a first choice for women of the reproductive age who want to preserve fertility. Therefore, an alternative method of treatment in this group of patients is myomectomy. It can be done by either laparotomy, minilaparotomy, hysteroscopy or laparoscopy or a combination of above mentioned ways.

The strict criteria for laparoscopic treatment do not exist but the size, location of myoma have to be taken into consideration. It is possible to differentiate 3 types of myomas, according to their location - subserosal (protruding outside the uterus), intramural (in the myometrium) and submucosal (projecting into the uterine cavity). Laparoscopic approach is employed in patients with subserosal or intramural fibroids with diameter less then $10 \mathrm{~cm}$ and their number not exceeding $5^{6}$. Generally, contraindications for LM include suspected malignancy and excessive size of a myoma ${ }^{7}$. For submucosal or numerous fibroids, hysteroscopic myomectomy is recommended ${ }^{8}$. Leiomyomas can be treated also with a nonsurgical and non-pharmacological approach like embolization of the uterine arteries. This method enables to achieve reduction of tumor volume and effectively alleviates symptoms. Procedure is indicated in woman who do not wish to be treated surgically but is not free of serious complications.

\section{Purpose}

Evaluation of feasibility, effectiveness and safety of LM in the treatment of large myomas.

\section{Materials and methods}

We performed research of Pubmed, Springer Link and ResearchGate databases. Studies analyzing methods of myomas' treatment were analized. 


\section{Results}

There are many advantages of laparoscopic myomectomy as compared to the classic myomectomy done by laparotomy. The main advantage is shorter hospitalization, less postoperative pain and reduced incidence of the risk of adhesions ${ }^{9}$. LM is indicated for small or medium size fibroids ${ }^{10}$ not exceeding $10 \mathrm{~cm}$ in diameter.

As it was mentioned, LM is considered as a challenging method especially in the cases of large myomas' removal. Difficulties include long duration of the surgery, risk of intraoperative bleeding and possible conversion to laparotomy. ${ }^{11}$ Meticulous presurgical imaging is necessary to detect any uterine malignancy. Unfortunately, currently there is no reliable method to differentiate benign from malignant leiomyomas (leiomyosarcomas or endometrial stromal sarcomas) ${ }^{12}$. Neither USG nor MRI does not predict tumor's malignancy, if there are no metastases or infiltrations. The assessment of angiogenesis by Doppler velocimetry technique seems to be helpful in diagnosing endometrial malignancy. Also a rapid postmenopausal growth can be a symptom of malignancy ${ }^{13}$. A complex assessment of clinical data and imaging is crucial in order to plan the treatment. Power morcellation can create a risk of neoplasm dissemination and of course is strictly contraindicated when a malignancy is suspected. However, the incidence of leiomyosarcomas is rare studies showed that only about $0.25 \%$ of women who undergo surgery for fibroids can have a leiomyosarcoma ${ }^{14}$. Next disadvantage of LM in large tumors is obliteration of vision due to the size of the myoma. Also, excessive bleeding should be taken into consideration, as the amount of bleeding during surgery for myomas larger than $10 \mathrm{~cm}$ increases. ${ }^{15}$

In some studies ${ }^{16}$, a GnRH therapy before LM was administered in order to reduce size of the fibroma and volume of intraoperative blood loss. However, the procedure is of unproven efficacy. What is more a degeneration of the fibroma can occur that added technical difficulties to the laparoscopic procedure.

The data concerning laparoscopic myomectomy in large fibroids is limited. The first prospective study was performed by Sinha $\mathrm{R}$ et al. and involved 51 women with at least one myoma of a large size. The study concluded that LM can be safely used alternatively to laparotomy in large myomas treatment ${ }^{17}$. Another study [1] suggested that LM has many advantages as a surgical method of treating fibroids. Aksoy et al. presented case report of patients with $17 \mathrm{~cm}$ myoma ${ }^{18}$. Tumor was removed successfully without any harm to the patient's endometrium. There is also a description of a successful LM in 34-year old woman's with $18 \mathrm{~cm}$ myoma $^{19}$. Finally, a research done by Hyo Jin Yoon et al. ${ }^{20}$ assessed the effectiveness of laparoscopy for large myomas. Fifty one patients were analyzed and study proved that procedure is feasible, there was no conversion to laparotomy and all of the fibromas were removed successfully. The largest diameter of the fibroma was $15.2 \mathrm{~cm}$. Seven patients required postoperative transfusion.

It is suggested that LM is an option not only for women, who want to retain their fertility but also for those, who are infertile because of the distortion of endometrial cavity by a myoma. LM enhances the postsurgical pregnancy rate ${ }^{21}$, which is comparable to the rate achieved with laparotomy ${ }^{22}$.

LM increases life quality and sexual functions when compared to laparotomy due to less trauma and postsurgical pain ${ }^{23}$. It was found that patients can return to work quicker if they undergo laparoscopic or robotic myomectomy, comparing to those who had laparotomy ${ }^{24}$. However it is suggested that favorable effects of LM could be short-term. The recurrence rate of fibroids is $15 \%$ and hysterectomy will be required for about $10 \%$ of women within 5 to 10 years after laparoscopic myomectomy ${ }^{25}$. Thus, a potentially successful approach can influence life quality and cause sexual impairment in the long term, however more investigations are needed. LM has positive impact on pregnancy outcomes and can be safely used in women, 
who want to retain fertility and become pregnant. Studies showed that pregnancy rate is exceeding $60 \%{ }^{26},{ }^{27}$. Laparoscopic myomectomy is linked to the increased risk of uterine rupture. However, the risk seems to be relatively small, the overall incidence was $0.07 \%{ }^{28}$ as compared to $0.012 \%$ for those without any previous uterine scar.

There is no one recommended mode of delivery after myomectomy therefore there is a need for more randomized studies. In some studies, it is advised that vaginal delivery can be achieved after LM if performed according to protocol of vaginal birth after cesarean section ${ }^{29}$. Contrary to the above, ACOG recommended a cesarean section after myomectomy The procedure should be performed in 38 week of pregancy ${ }^{30}$.

\section{Discussion}

The management of myomas relies mostly on the surgical techniques, both classic and minimally invasive. Although LM is more feasible in myomas of smaller size (up to $10 \mathrm{~cm}$ ), literature suggests that this method can be successfully used in the treatment of larger myomas. LM for large tumors is widely considered as a challenging method. The potential complications of this methods includes excessive bleeding, prolonged surgery, conversion to laparotomy, injuries to urinary or digestive tracts. Articles published from 1993 to 2013 reported 55 complications related to the use of power morcellator. They included large bowel, kidney, the bladder injuries, injuries of the diaphragm and major vessel. Six death occurred ${ }^{31}$. Overall, the frequency of injuries to the organs caused by power morcellator was assessed as $(0.12 \%)^{32}$. Undoubtedly, LM has many advantages when compared to laparotomy. They include less pain and faster postsurgical recovery ${ }^{33}$. However, because of the possible complications, the treatment options of large myomas are not standardized and the patient's approach could be complex.

When uterus preservation is demanded LM allows to retain fertility. Other advantages of LM involve faster recovery, shorter hospitalization, less pain and decreased risk of postsurgical adhesions. Data concerning use of LM in the removal of large myomas is limited but safety and efficacy of the method seems to be proven.

\section{Conclusions}

Removal of the large myomas by laparoscopy is still regarded as a surgical and technical challenge. The LM removal of a large tumor can be recommended only following balanced assessment of all of possible risks and technical difficulties. The main factors behind surgical success are the experience and skills of the surgeon.

\section{Bibliography}

${ }^{1}$ Aksoy, H., Aydin, T., Özdamar, Ö. et al. Successful use of laparoscopic myomectomy to
remove a giant uterine myoma: a case report. J Med Case Reports 9, 286 (2015).
https://doi.org/10.1186/s13256-015-0771-9

${ }^{2}$ Sano R, Suzuki S, Shiota M. Laparoscopic Myomectomy for the Removal of Large Uterine Myomas. Surg J (N Y). 2019;6(Suppl 1):S44-S49. Published 2019 Sep 11. doi:10.1055/s0039-1694989

3 Sinha, Rakesh \& Sundaram, Meenakshi. (2009). Laparoscopic Management of Large Myomas. Journal of gynecological endoscopy and surgery. 1. 73-82. 10.4103/09741216.71611 . 
${ }^{4}$ Mettler L, Schollmeyer T, Tinelli A, Malvasi A, Alkatout I. Complications of uterine fibroids and their management, surgical management of fibroids, laparoscopy and hysteroscopy versus hysterectomy, haemorrhage, adhesions, and complications. Obstet Gynecol Int. 2012;2012:791248

${ }^{5}$ Bouchard P, Chabbert-Buffet N, Fauser BC. Selective progesterone receptor modulators in reproductive medicine; pharmacology, clinical efficacy and safety. Fertil Steril2011;96:11751189.

${ }^{6}$ Takeuchi, Hiroyuki \& Kuwatsuru, Ryohei. (2003). The Indications, Surgical Techniques, and Limitations of Laparoscopic Myomectomy. JSLS : Journal of the Society of Laparoendoscopic Surgeons / Society of Laparoendoscopic Surgeons. 7. 89-95.

7 Dubuisson JB, O'Leary T, Feki A, Bouquet DE Jolinière J, Dubuisson J. Laparoscopic myomectomy. Minerva Ginecol. 2016;68(3):345-351.

8 Pakrashi T. New hysteroscopic techniques for submucosal uterine fibroids. Curr Opin Obstet Gynecol. 2014;26(4):308-313. doi: 10.1097/GCO.0000000000000076.

9 Hurst BS, Mathews ML, Marshburn PB. Laparoscopic myomectomy for symptomatic uterine myomas. Fertil Steril. 2005;83:1-23

10 Takeuchi H, Kuwatsuru R. The indications, surgical techniques, and limitations of laparoscopic myomectomy. JSLS. 2003;7(2):89-95

${ }^{11}$ Dubuisson J-B, Fauconnier A, Babaki-Fard K, Chapron C. Laparoscopic myomectomy: a current view. Hum Reprod Update. 2000;6:588-94

12 Vilos GA, Allaire C, Laberge PY, Leyland N; SPECIAL CONTRIBUTORS. The management of uterine leiomyomas. J Obstet Gynaecol Can. 2015;37(2):157-178. doi:10.1016/S1701-2163(15)30338-8

${ }^{13}$ Maizlin Z., Vos P., Cooperberg P. Ist it e fibroid? Are you sure? Sonography with MRI assistance. Ultrasound Quarterly, 2007: 55-62

${ }^{14}$ Knight J, Falcone T. Tissue extraction by morcellation: a clinical dilemma. J Min Invas Gynecol 2014;21:319-20.

15 Takeuchi, Hiroyuki \& Kuwatsuru, Ryohei. (2003). The Indications, Surgical Techniques, and Limitations of Laparoscopic Myomectomy. JSLS : Journal of the Society of Laparoendoscopic Surgeons / Society of Laparoendoscopic Surgeons. 7. 89-95.

${ }^{16}$ Sebastiano Campo, Nicola Garcea, Laparoscopic myomectomy in premenopausal women with and without preoperative treatment using gonadotrophin-releasing hormone analogues, Human Reproduction, Volume 14, Issue 1, January 1999, Pages 4448, https://doi.org/10.1093/humrep/14.1.44

${ }^{17}$ Sinha R, Hegde A, Warty N, Patil N. Laparoscopic excision of very large myomas. J Am Assoc Gynecol Laparosc. 2003;10(4):461-468. doi:10.1016/s1074-3804(05)60145-4 
${ }^{18}$ Aksoy, H., Aydin, T., Özdamar, Ö. et al. Successful use of laparoscopic myomectomy to remove a giant uterine myoma: a case report. J Med Case Reports 9, 286 (2015). https://doi.org/10.1186/s13256-015-0771-9

${ }^{19}$ Laparoscopic myomectomy of a giant myoma. Kavallaris A, Zygouris D, Chalvatzas N, Terzakis E. Clin Exp Obstet Gynecol. 2013;40(1):178-80. PMID: 23724541

20 Yoon, Hyo \& Kyung, Min \& Jung, Un \& Choi, Joong Sub. (2007). Laparoscopic Myomectomy for Large Myomas. Journal of Korean medical science. 22. 706-12. 10.3346/jkms.2007.22.4.706.

${ }^{21}$ Kumakiri J, Takeuchi H, Kitade M, Kikuchi I, Shimanuki H, Itoh S, et al. Pregnancy and delivery after laparoscopic myomectomy. J Minim Invasive Gynecol. 2005;12:241-6.

${ }^{22}$ Seracchioli R, Rossi S, Govoni F, Rossi E, Venturoli S, Bulletti C, et al. Fertility and Obstetric outcome after laparoscopic myomectomy of large myomata: A randomized comparison with abdominal myomectomy. Hum Reprod. 2000;15:2663-8.

${ }^{23}$ Radosa, Julia \& Radosa, Christoph \& Mavrova, Russalina \& Wagenpfeil, Stefan \& Hamza, Amr \& Joukhadar, Ralf \& Baum, Sascha \& Karsten, Maria \& Juhasz-Böss, Ingolf \& Solomayer, Erich-Franz \& Radosa, Marc. (2016). Postoperative Quality of Life and Sexual Function in Premenopausal Women Undergoing Laparoscopic Myomectomy for Symptomatic Fibroids: A Prospective Observational Cohort Study. PLOS ONE. 11. e0166659. 10.1371/journal.pone.0166659.

${ }^{24}$ Griffin L, Feinglass J, Garrett A, Henson A, Cohen L, Chaudhari A, et al. Postoperative outcomes after robotic versus abdominal myomectomy. JSLS, 2013; 17: 407-13. $10.4293 / 108680813 X 13693422521557$

${ }^{25}$ Garcia CR. Management of the symptomatic fibroid in women older than 40 years of age: hysterectomy or myomectomy? Obstet Gynecol Clin North Am 1993;20:337-48.

${ }^{26}$ G. Favero, "Tips and tricks for successful manual morcellation: A response to "vaginal morcellation: A new strategy for large gynecological malignant tumors extraction. A pilot study"," Gynecologic Oncology, vol. 128, no. 1, p. 151, 2013.

${ }^{27}$ L. Mettler, T. Schollmeyer, A. Tinelli, A. Malvasi, and I. Alkatout, "Complications of uterine fibroids and their management, surgical management of fibroids, laparoscopy and hysteroscopy versus hysterectomy, haemorrhage, adhesions, and complications," Obstetrics and Gynecology International, vol. 2012, Article ID 791248, 8 pages, 2012.

${ }^{28}$ Pakniatp, Hamideh \& Soofizadehp, Nasrin \& Khezri, Marzeih. (2016). Spontaneous uterine rupture after abdominal myomectomy at the gestational age of 20 weeks in pregnancy: A case report. Int J Reprod BioMed. 14. 483-486. 10.29252/ijrm.14.7.8.

${ }^{29}$ Kumakiri, Jun \& Takeuchi, Hiroyuki \& Kitade, Mari \& Kikuchi, Iwaho \& Shimanuki, Hiroto \& Itoh, Shigeru \& Kinoshita, Katsuyuki. (2003). Pregnancy and delivery after laparoscopic myomectomy. Journal of minimally invasive gynecology. 12. 241-6. 10.1016/j.jmig.2005.03.011. 
${ }^{30}$ Obstetrics \& Gynecology: April 2004 - Volume 103 - Issue 4 - p 817-818

${ }^{31}$ Milad M. P., Milad E. A. Laparoscopic morcellator-related complications. Journal of Minimally Invasive Gynecology. 2014;21(3):486-491. doi: 10.1016/j.jmig.2013.12.003.

${ }^{32}$ Tanos V, Berry KE, Frist M, Campo R, DeWilde RL. Prevention and Management of Complications in Laparoscopic Myomectomy. Biomed Res Int. 2018;2018:8250952. Published 2018 Mar 5. doi:10.1155/2018/8250952

33 Jin $\mathrm{C}$, Hu Y, Chen XC, Zheng FY, Lin F, Zhou K, et al. Laparoscopic versus open myomectomy - a meta-analysis of randomized controlled trials. Eur J Obstet Gynecol Reprod Biol 2009;145:14-21. 